\title{
Robust Inventory Financing Model with Partial Information
}

\author{
Yong Wang, ${ }^{1}$ Jixiang Zhou, ${ }^{1}$ Hailei Sun, ${ }^{1}$ and Lin Jiang ${ }^{2}$ \\ ${ }^{1}$ School of Economics and Business Administration, Chongqing University, Chongqing 400044, China \\ ${ }^{2}$ School of Mathematics and Information Engineering, Chongqing University of Education, Chongqing 400067, China \\ Correspondence should be addressed to Yong Wang; wangyongkt@163.com
}

Received 15 January 2014; Revised 30 April 2014; Accepted 19 May 2014; Published 11 June 2014

Academic Editor: Vincent Charles

Copyright (C) 2014 Yong Wang et al. This is an open access article distributed under the Creative Commons Attribution License, which permits unrestricted use, distribution, and reproduction in any medium, provided the original work is properly cited.

\begin{abstract}
Given current fast-changing market conditions and difficulty in obtaining financing for small- and medium-sized enterprises, this paper studies the robust inventory financing model under partial information, that is, where the demand distribution is partly known. Two demand information cases are discussed: (1) the mean and variance and (2) the support of the demand distribution. In this setting, the robust method that maximizes the worst-case profit and minimizes the firm's maximum possible regret of not acting optimally would be used to formulate the optimal sales quantity. We show that the approach used in this paper is tractable, and we provide an explicit expression for the robust optimal policy. We then use numerical examples to compare the firm's losses under two demand information cases with those occurring under demand certainty. More importantly, the numerical examples indicate that our robust inventory financing model can obtain a robust but not conservative solution.
\end{abstract}

\section{Introduction}

The topic of this paper is inspired by the challenges in obtaining financing faced by small- and medium-sized enterprises. They often have little in the way of the fixed assets that lenders are usually willing to recognize as security for loans or leases, so it is difficult for them to borrow money except at prohibitive interest rates [1]. Even if a loan is taken out under such terms, the firm's development will be further constrained due to lack of money. To alleviate such problems, the practice of inventory financing has become increasingly common. Inventory financing allows firms to use their inventory resources as pledges to obtain loans from a bank. After examination and approval, the bank will transfer the inventory to a third-party logistics (3PL) provider who will store and monitor the inventory. The bank then lends the firm money at a previously agreed interest rate [2]. By using inventory financing, the bank, 3PL, and the borrowing company cooperate in a new platform to achieve a winwin-win situation. This practice has seen rapid growth in China in recent years and is now very popular. It can also be seen to be operating informally in the practice of some small- and medium-sized enterprises to mortgage part of their selling products to obtain loans to carry out different profitable projects. For example, in Shandong province, some enterprises that sell fodder for chicken, pigs, or other animals use their inventory as pledges to obtain loans, which they then use for breed aquatics. In Hebei province, a steel plant mortgaged part of its products to obtain loans to develop a new product.

Although inventory financing is widely used, research about it remains scant. Jokivuolle and Peura [3] present a model of risky debt in which collateral value is correlated with the possibility of default. Cossin et al. [4] determine the default probability of enterprises exogenously and acquire a determination model of the collateral haircut consistent with the bank's level of risk tolerance based on the reducedform approach. Babich et al. [5] study firm procurement with financing decisions on loan amount from each source. In contrast to Babich et al. [5], Li et al. [6] assume that a firm can continue operation by paying a default penalty even after it defaults on a loan for a particular business activity. Dada and $\mathrm{Hu}$ [7] present a capital-constrained newsvendor model wherein the newsvendor must borrow money from 
a bank to finance additional procurement. In their paper, they assume that the bank is coordinated and strategic regarding the newsvendor's order quantity as a Stackelberg leader.

All of these researches, however, focus on the deterministic case. But, the real world is full of uncertainties. As the number of sources of uncertainty increases, studies on uncertainty are also increasing, such as the researches carried out by Suo et al. [8], Xu [9], and Lari [10]. Furthermore, some researchers have studied inventory financing problems under demand uncertainty. Gupta and Wang [11] present a discrete time model of a retailer's operations with random demand to prove that the optimal policy's structure is not affected by credit terms. Buzacott and Zhang [1] model the available cash in each period as a function of assets and liabilities that may be updated periodically according to the dynamics of production activities. Lee and Rhee [12] investigate the impact of inventory financing costs on supply chain coordination.

However, all of the above researches on inventory financing mainly focus on scenarios in which complete information about demand is available. In practice, it is often difficult to completely characterize demand or the demand distribution, especially in fast-changing markets. Accordingly, in this paper, we study the inventory financing problem with only partial information available regarding the demand distribution, and two different types of demand information are discussed. Based on this, we propose a robust inventory financing model, and rather than giving an exact distribution, we limit the information to only know the mean and variance or the support. From this, we derive the robust optimal sales quantity and the profit or loss under the two different types of demand information. The contributions of this paper are as follows.

(1) We combine inventory financing with sales. Furthermore, we jointly consider the impacts of inventory financing and project investment on a firm's decisions and profit.

(2) We introduce partial demand information into the inventory financing problem. Furthermore, the impacts of two types of partial demand information (mean and variance and support) on the firm's decisions and profit are considered.

The remainder of this paper is organized as follows. In Section 2, we describe the inventory financing model and introduce the notations. In Section 3, we discuss the inventory financing model with partial demand information (mean and variance). In Section 4, we formulate the inventory financing model with partial demand information (support). In Section 5, we present the numerical results. In Section 6, we conclude the results and suggest topics for future research.

\section{The Model}

We construct a single-period model to study a firm's inventory financing decisions. In our model, a firm that faces a random demand in the market and that possesses partial information about such demand faces a trade-off between pledging for cash to invest and selling before the sales season. The pledges will be taken care of by a designated third party and the unsold product will be kept as inventory by the firm. Consider the time period $[0, T]$; for simplicity, we assume that the investment horizon and the financing horizon are both $[0, T]$. At time 0 , the firm has no cash and must make a choice between pledging some of her products to invest and selling all of them. If the firm decides to pledge, it must further decide how many. The related notations are given as follows:

$p$ : the unit selling price;

$d$ : the demand in the single period, a random variable; $c_{1} ; c_{2}$ : the unit storage cost; the unit penalty cost for shortages;

Q: the initial inventory;

$q ; Q-q$ : the sales quantity, the decision variable; the pledge quantity;

$A S, B S$ : the revenue if investment succeeded and initial investment is $S$, the revenue if investment failed and initial investment is $S, A \geq 1,0 \leq B<1$;

$\alpha, 1-\alpha$ : the probability of success of the investment and the probability of failure of the investment;

$\lambda$ : loan-to-value ratios specified in the contract with the bank;

$i$ : the simple interest rate;

$L+c_{3}(Q-q)$ : where $L$ is fixed service fee paid to the $3 \mathrm{PL} ; c_{3}$ is the unit service cost.

The expected profit for the firm in the single period is

$$
\begin{aligned}
& E[\pi(q)] \\
& =E\left[p \min \{q, d\}-c_{1}(q-d)^{+}-c_{2}(d-q)^{+}\right. \\
& +\alpha A \lambda p(Q-q)+(1-\alpha) B \lambda p(Q-q) \\
& \left.-\lambda p(Q-q)-i \lambda p(Q-q) T-L-c_{3}(Q-q)\right] \text {. }
\end{aligned}
$$

\section{Inventory Financing Decision with Partial Demand Information (Mean and Variance)}

In this section, we assume that the firm possesses only partial demand information, that is, mean $\mu$ and variance $\sigma^{2}$, and its objective is to maximize its expected profit. Under the above conditions, the robust optimization approach first addressed by Scarf [13] will be adopted to model demand uncertainty. Accordingly, the firm's objective can be rewritten as follows:

$$
\max _{q} \inf _{d \sim\left(\mu, \sigma^{2}\right)} E[\pi(q)]
$$


Since $\min \{q, d\}=d-(d-q)^{+}$and $(q-d)^{+}=(q-d)+$ $(d-q)^{+}$, we obtain

$$
\begin{aligned}
E[\pi(q)]= & p \mu-\left(p+c_{1}+c_{2}\right) E\left[(d-q)^{+}\right]-c_{1}(q-\mu) \\
& +\alpha A \lambda p(Q-q)+(1-\alpha) B \lambda p(Q-q) \\
& -\lambda p(Q-q)-i \lambda p(Q-q) T-L-c_{3}(Q-q) .
\end{aligned}
$$

Following Gallego and Moon [14], we get

$$
f(q)=\inf _{D \sim\left(\mu, \sigma^{2}\right)} E[\pi(q)]=\Psi(q)-\Phi(q),
$$

where

$$
\begin{aligned}
& \Psi(q)= p \mu-c_{1}(q-\mu)+\alpha A \lambda p(Q-q) \\
&+(1-\alpha) B \lambda p(Q-q) \\
&-\lambda p(Q-q)-i \lambda p(Q-q) T-L-c_{3}(Q-q) \\
& \Phi(q)=\left(p+c_{1}+c_{2}\right) \frac{\sqrt{\sigma^{2}+(q-\mu)^{2}}-(q-\mu)}{2}
\end{aligned}
$$

Note that (5) indicates the riskless profit function (e.g., Mills [15] and Petruzzi and Dada [16]), the profit in the certainty equivalent problem in which $d$ is replaced by $\mu$. Equation (6) indicates the loss function [17] because of the uncertainty in demand. Thus, from (4), we can find that (1) the expected profit with uncertainty is less than that with certainty and (2) we can increase the expected profit through decreasing uncertainty. Therefore, the objective is transformed to

$$
\max _{q} f(q) .
$$

We take the first and second partial derivatives of $f(q)$ with respect to $q$ :

$$
\begin{aligned}
\frac{\partial f(q)}{\partial q}= & -\alpha A \lambda p-(1-\alpha) B \lambda p+\lambda p+i \lambda p T+c_{3} \\
& +\frac{1}{2}\left(p-c_{1}+c_{2}\right) \\
& -\frac{1}{2}\left(p+c_{1}+c_{2}\right) \frac{q-\mu}{\sqrt{\sigma^{2}+(q-\mu)^{2}}}, \\
\frac{\partial^{2} f(q)}{\partial q^{2}}= & -\frac{1}{2}\left(p+c_{1}+c_{2}\right) \frac{\sigma^{2}}{\left[\sigma^{2}+(q-\mu)^{2}\right]^{3 / 2}} .
\end{aligned}
$$

Notice from (9) that $f(q)$ is concave in $q$. Therefore, we can obtain the optimal sales quantity through setting $\partial f(q) / \partial q=$ 0 .

Proposition 1. The optimal sales quantity is given by

$$
q^{*}=\frac{E}{\sqrt{G^{2}-E^{2}}} \sigma+\mu,
$$

where $E=-\alpha A \lambda p-(1-\alpha) B \lambda p+\lambda p+i \lambda p T+c_{3}+(1 / 2)(p-$ $\left.c_{1}+c_{2}\right), G=(1 / 2)\left(p+c_{1}+c_{2}\right)$. To examine the effects of certain exogenous variables on sales quantity, we differentiate $q$ with respect to $T, i, \lambda, c_{1}, c_{2}, c_{3}$ and obtain the following results.

Proposition 2. (1) Consider $d q / d T>0, d q / d i>0$;

(2) $d q / d \lambda>0$, when $-\alpha A \lambda p-(1-\alpha) B \lambda p+\lambda p+i \lambda p T>0$, $d q / d \lambda<0$, when $-\alpha A \lambda p-(1-\alpha) B \lambda p+\lambda p+i \lambda p T<0$;

(3) $d q / d c_{1}<0, d q / d c_{2}>0, d q / d c_{3}>0$.

Proposition 2(1) indicates that $q$ increases with increasing $T$ or $i$. This is straightforward because whether there is an increase in $T$ or $i$, both mean an increase in the cost of loan. Therefore, the firm will increase the sales quantity and decrease the pledge quantity. Proposition 2(2) shows that when the expected revenue from an investment is lower than the cost of the loan, that is, the firm suffers loss from the investment, then the firm will increase the sales quantity and decrease the pledge quantity. Accordingly, when the expected revenue of an investment is higher than the cost of the loan, the firm will decrease the sales quantity. Proposition 2(3) indicates that $q$ decreases as $c_{1}$ increases and also increases with increasing $c_{2}$ or $c_{3}$. As when $c_{1}$ increases, the cost of inventory will also increase, so the firm will decrease the quantity of inventory, and when $c_{2}$ or $c_{3}$ increases, the cost of pledges will increase, so the firm will increase the quantity of inventory; that is, it will decrease the amount of pledges. We can find a simple truth from the above description; that is, the firm will increase the quantity of inventory if the cost of pledges increases, and it will decrease the quantity of inventory when the cost of inventory increases.

\section{Inventory Financing Decision with Partial Demand Information (Support)}

Facing a rapidly changing market, a firm might not have past sales data available, or the data might even be absent, in particular for products with short life cycles in new markets. Sometimes, only an educated guess is available for the lower and upper bounds of demand. In such a situation, the approaches based on Bayesian learning [18] or nonparametric learning $[19,20]$ are not applicable. Furthermore, some researchers think that the maximin approach is too conservative. Therefore, the minimax regret, a less conservative approach provided by Savage [21], will be adopted in this section. The minimax regret has also been investigated by Vairaktarakis [22], Yue et al. [23], Perakis and Roeis [24], and Lin and $\mathrm{Ng}$ [25].

In this section, we assume that a firm only knows the support of demand $[M, N]$ and its objective is to minimize its regret. To guarantee the probability of pledges, we assume that $Q>N$. Let $z$ denote the optimal sales quantity. For a given decision $q$ and a probability distribution $F$, the regret measures the additional profit that could have been obtained if the firm possessed full information about the distribution; that is, $\max _{z \in[M, N]} E\left[\pi_{F}(z)\right]-E\left[\pi_{F}(q)\right]$. The maximum regret $\rho(q)=\max _{F} \max _{z \in[M, N]} E\left[\pi_{F}(z)\right]-E\left[\pi_{F}(q)\right]$ can be regarded as the maximum price one would pay to know the exact 
demand distribution (e.g., with a marketing survey) [24]. Therefore, the decision criterion consisting of minimizing $\rho(q)$ can be written as follows:

$$
\begin{aligned}
\rho^{*} & =\min _{q \in[M, N]} \rho(q) \\
& =\min _{q \in[M, N]} \max _{F} \max _{z \in[M, N]} E\left[\pi_{F}(z)\right]-E\left[\pi_{F}(q)\right] .
\end{aligned}
$$

We formulate the problem of identifying the worst-case demand scenario as a moment problem. Then, by inverting the order of maximization, (11) can be rewritten as follows:

$$
\begin{aligned}
& \min _{q \in[M, N]} \rho(q) \\
& =\min _{q \in[M, N]} \max _{z \in[M, N]}\left\{\max _{F} E\left[\pi_{F}(z)\right]-E\left[\pi_{F}(q)\right]\right\} \\
& =\min _{q \in[M, N]} \max _{z \in[M, N]}\left\{\max _{F} \int_{M}^{N} p(\min \{z, d\}\right. \\
& \quad-\min \{q, d\}) d F(d)\} \\
& -\left(c_{1}+c_{2}\right)\left[(z-d)^{+}-(q-d)^{+}\right]+H(z-q),
\end{aligned}
$$

where $H=c_{2}-\alpha A \lambda p-(1-\alpha) B \lambda p+\lambda p+i \lambda p T+c_{3}$. Solving the programming problem (12), we obtain the optimal sales quantity.

Proposition 3 (see $[22,24]$ ). If the demand distribution is nonnegative, then, with support $[M, N]$, the optimal sales quantity in minimax regret is equal to the following:

$$
q_{R}^{*}=\frac{(p+H) N+\left(c_{1}+c_{2}-H\right) M}{p+c_{1}+c_{2}} .
$$

Proof. The inner problem of (12), consisting of finding the distribution that maximizes the regret for given $q$ and $z$, can be rewritten as follows:

$$
\begin{gathered}
\max _{F} \int_{M}^{N} p(\min \{d, z\}-\min \{d, q\}) d F(d)-\left(c_{1}+c_{2}\right) \\
\times\left[(z-d)^{+}-(q-d)^{+}\right]+H(z-q)
\end{gathered}
$$

s.t. $\quad d F(d) \geq 0$

$$
\int_{M}^{N} d F(d)=1 .
$$

By strong duality, problem (14) is equivalent to the following dual problem $([24,26])$ :

$$
\begin{array}{ll}
\min & 1 \cdot y \\
\text { s.t. } & 1 \cdot y \geq p(\min \{d, z\}-\min \{d, q\}) \\
& -\left(c_{1}+c_{2}\right)\left[(z-d)^{+}-(q-d)^{+}\right]+H(z-q) .
\end{array}
$$

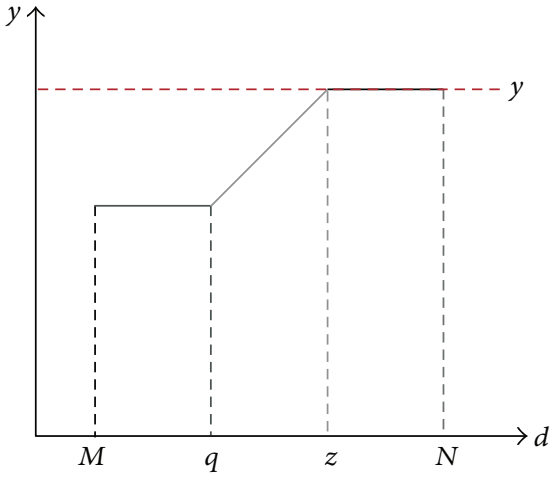

Figure 1: The case of $z \geq q$.

We distinguish the two cases $z \geq q$ and $z<q$ to discuss the programming problem (15). In order to reflect the two kinds of situations, we refer to the following two figures. Figure 1 reflects the case of $z \geq q$; accordingly, Figure 2 reflects the other case. From Figure 1, we can find that the minimum for $y$ in the case of $z \geq q$ is $(p+H)(z-q)$. And from Figure 2, the minimum for $y$ in the other case is $\left(H-c_{1}-c_{2}\right)(z-q)$. Therefore, we can obtain

$$
\begin{array}{rl}
\max _{F} & E\left[\pi_{F}(z)\right]-E\left[\pi_{F}(q)\right] \\
& = \begin{cases}(p+H)(z-q), & \text { if } z \geq p \\
\left(H-c_{1}-c_{2}\right)(z-q), & \text { if } z<q .\end{cases}
\end{array}
$$

Since $z \in[M, N]$, we can get

$$
\rho(q)=\left\{\begin{array}{c}
(p+H)(N-q), \\
\quad \text { if } q \leq \frac{(p+H) N+\left(c_{1}+c_{2}-H\right) M}{p+c_{1}+c_{2}} \\
\left(H-c_{1}-c_{2}\right)(M-q), \\
\quad \text { if } q>\frac{(p+H) N+\left(c_{1}+c_{2}-H\right) M}{p+c_{1}+c_{2}} .
\end{array}\right.
$$

Due to $q \in[M, N]$, then we obtain $\rho^{*}=1 /\left(p+c_{1}+c_{2}\right)\left(c_{1}+\right.$ $\left.c_{2}-H\right)(p+H)(N-M)$ and the optimal quantity $q_{R}^{*}=((p+$ $\left.H) N+\left(c_{1}+c_{2}-H\right) M\right) /\left(p+c_{1}+c_{2}\right)$.

Differentiating $q_{R}^{*}$ with respect to $T, i, \lambda, c_{1}, c_{2}, c_{3}$, we can then obtain the same conclusions as obtained in Section 3.

\section{Numerical Simulation}

In this section, based on our previous analysis, we will analyze the effects of different exogenous variable values on the firm's optimal decision and expected profit. We will then compare the results of the two demand information scenarios. We use the following parameters as benchmarks for the coming numerical studies:

$p=30, Q=1600, c_{1}=6, c_{2}=4, i=0.06, T=1, \lambda=0.75$, $A=1.5, B=0.5, \alpha=0.5, L=10, c_{3}=2, \mu=1000, \sigma=20$, $M=800, N=1200$.

Substituting the above data into (10) and (13), we obtain the optimal sales quantity $q^{*}=1035$ and $q_{R}^{*}=1174$. 


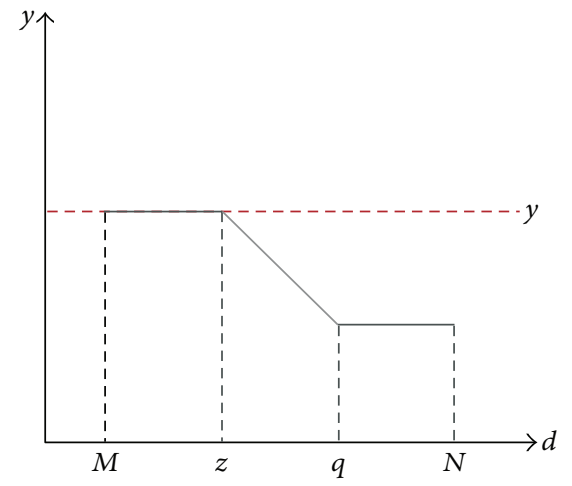

Figure 2: The case of $z<q$.

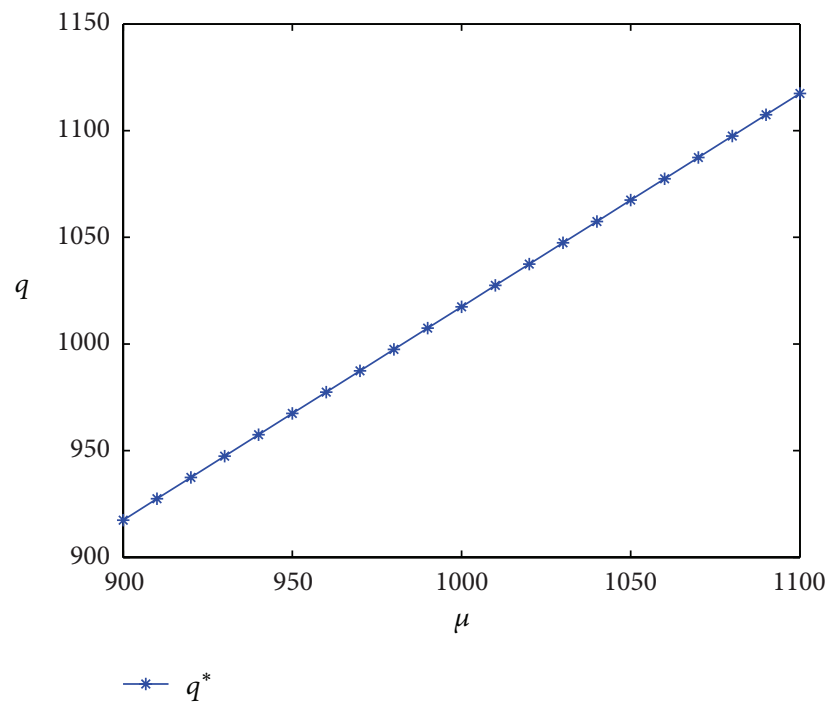

FIgURE 3: Impacts of $\mu$ on $q^{*}$.

Therefore, in the two cases, the firm will use some of its products for pledges, with the remainder being used for sales.

The impacts of having only partial demand information on the firm's decisions and the profit under two cases are shown in Figures 3, 4, 5, 6, 7, and 8. Figure 3 shows that, as $\mu$ increases, the firm should increase the sales quantity. This can be considered as an intuitive response because increasing the expected demand will force the firm to allocate more products to sales. More specifically, if $\mu$ increases by $10 \%$, the sales quantity should increase by $10 \%$. From Figure 4 , we find that a change of $\sigma$ has less impact on $q^{*}$. For instance, when $\sigma$ increases by $10 \%$, the quantity allocated for sales only increases by $0.3 \%$. Figure 5 indicates that increasing $\mu$ will enable the firm to earn higher profits. However, increasing $\sigma$ will have the opposite effect (see Figure 6). Furthermore, we also find that changing $\mu$ has a great impact on the firm's profit. For instance, when $\mu$ increases by $10 \%$, the firm's profit will increase by $12 \%$. However, when $\sigma$ increases by $10 \%$, the firm's profit will decrease by $0.07 \%$. On the basis of the above analysis, we conclude that, regardless of the firm's optimal sales quantity or the profit level, $\mu$ has a greater impact and the impact of $\sigma$ can be omitted. Therefore, we suggest the

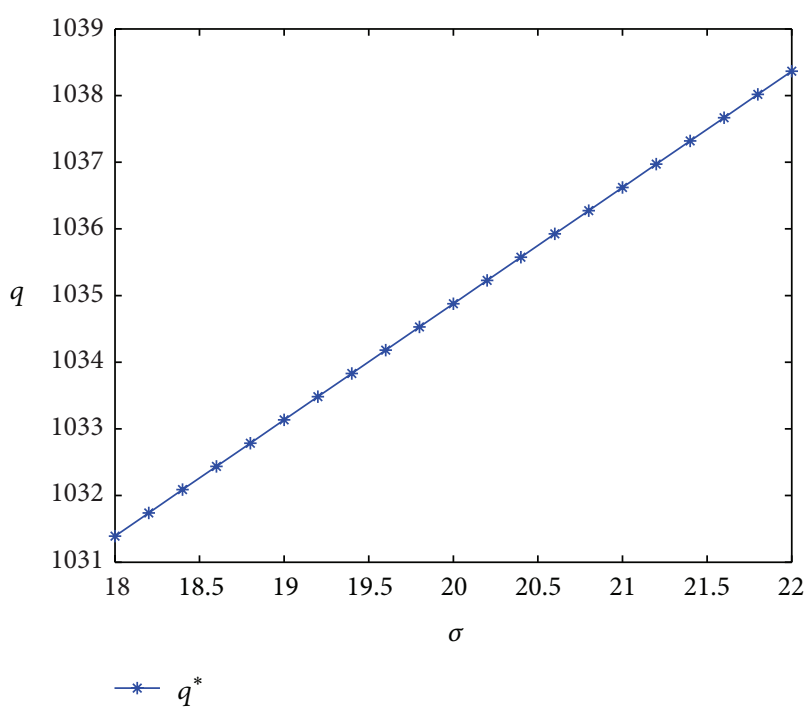

FIGURE 4: Impacts of $\sigma$ on $q^{*}$.

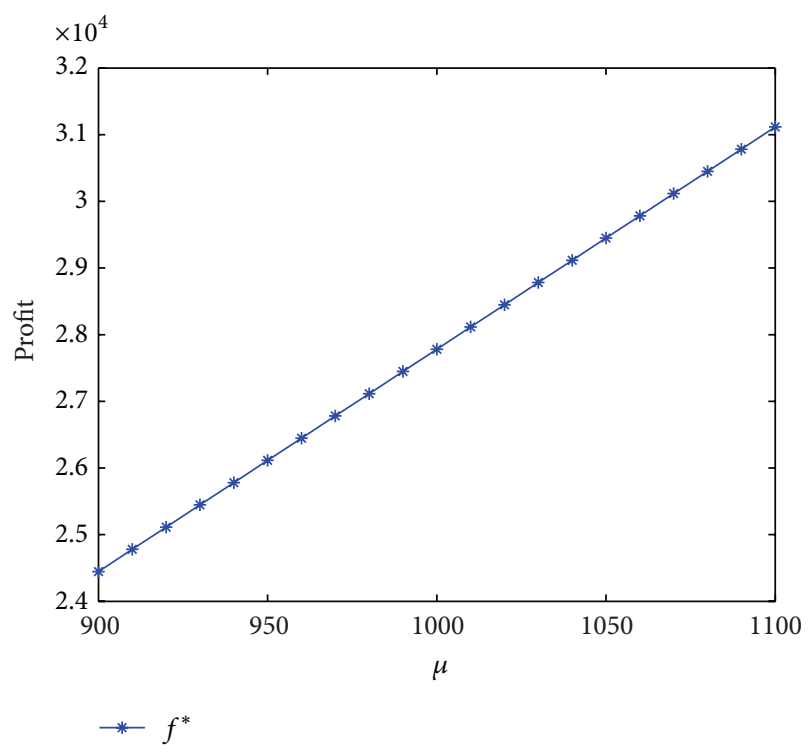

Figure 5: Impacts of $\mu$ on $f^{*}$.

firm should invest more for the information of the mean of the demand and less for the information of the variance of the demand. From Figures 7 and 8, we can see that a positive correlation exists between $M$ (or $N$ ) and $q_{R}^{*}$. In other words, $q_{R}^{*}$ increases with increasing $M$ (or $N$ ). However, the impacts of $N$ on $q_{R}^{*}$ are much greater than $M$. For instance, when $N$ increases by $10 \%, q_{R}^{*}$ increases by $9.5 \%$; if $M$ increases by $10 \%$, then $q_{R}^{*}$ only increases by $0.5 \%$. If the firm only cares about the maximum price $\rho^{*}$ it pays to know the exact demand distribution, then it only needs to know the absolute difference between $M$ and $N$, which can be found from the expression of $\rho^{*}$.

The impacts of $T, \alpha$, and $\lambda$ on $q^{*}$ and $q_{R}^{*}$ under the two cases are shown in Figures 9, 10, and 11. Figure 9 shows that, as described previously, as $T$ increases, the firm will raise 


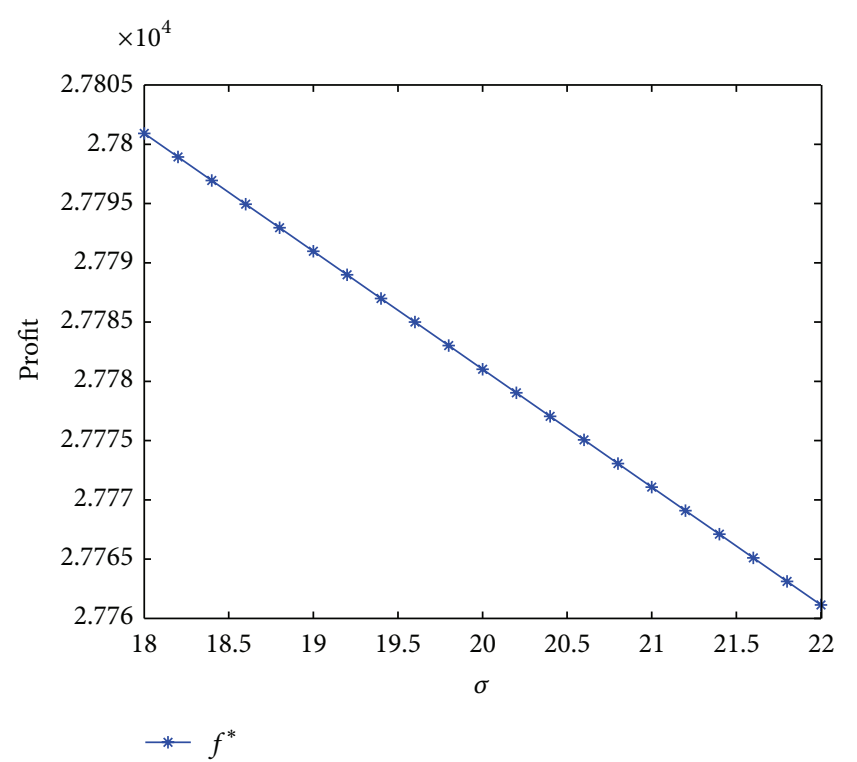

Figure 6: Impacts of $\sigma$ on $f^{*}$.

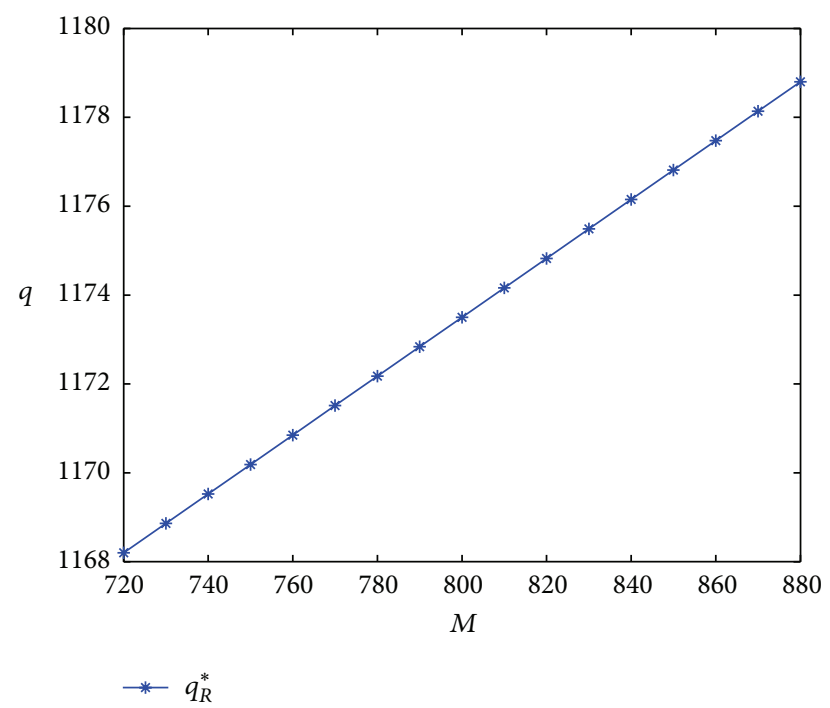

Figure 7: Impacts of $M$ on $q_{R}^{*}$.

the sales quantity in two cases; that is, it will reduce the pledge quantity. Figure 10 indicates that the firm's optimal sales quantities $q^{*}$ and $q_{R}^{*}$ decrease with increasing $\alpha$. This is straightforward because an increase in $\alpha$ intuitively means an increase in expected income from the investment, so the firm will pledge more products for money; that is, the quantity for sales will decrease. The conclusion we obtain from Figure 11 is consistent with that stated in Proposition 2(2); that is, when the expected revenue from an investment is lower than the cost of loan, the firm will increase the sales quantity to reduce borrowing costs. From Figures 3, 4, 5, 6, 7, 8, 9, 10, and 11, we can also find that no matter how $T, \alpha, \lambda$ change, $q^{*}<q_{R}^{*}$. This reflects the fact that the minimax regret is a less conservative approach. Therefore, when a firm only knows the support of demand, it will distribute more products for sales.

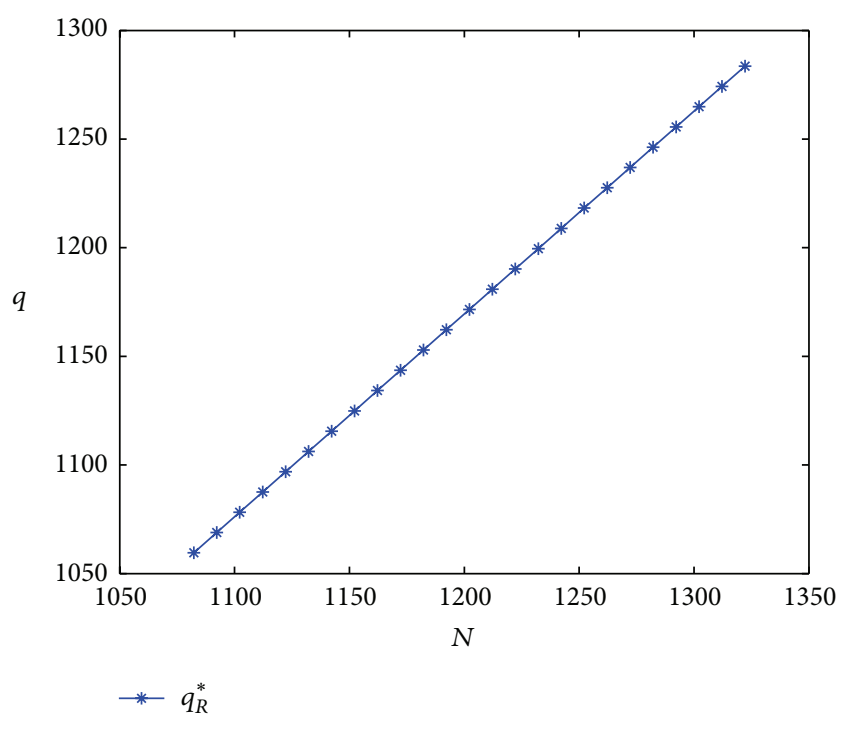

FIgURE 8: Impacts of $N$ on $q_{R}^{*}$.

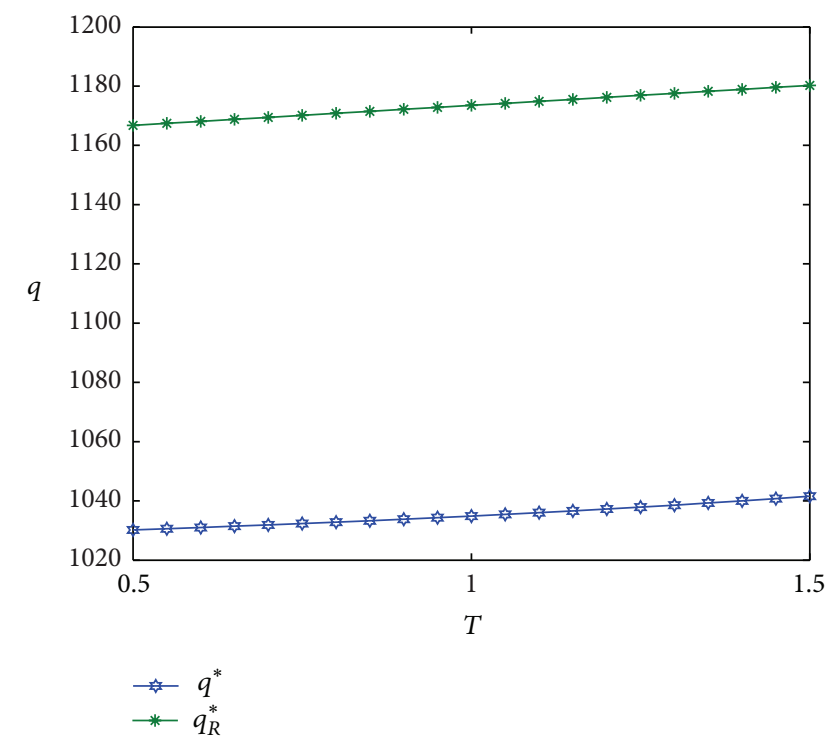

Figure 9: Impacts of $T$ on $q^{*}$ and $q_{R}^{*}$.

To verify the robustness of our methods, we will first make a comparison between the profits under demand certainty and uncertainty (with $\mu$ and $\sigma$ ), and then we will investigate the effects of incomplete information upon the two approaches. In accordance with (4)-(6), we can calculate the firm's profit under both demand certainty and uncertainty. The impacts of $T, \alpha$, and $\lambda$ on profits under demand certainty and uncertainty (with $\mu$ and $\sigma$ ) are shown in Figures 12, 13, and 14, and the losses of the two approaches due to partial information under different values of $T, \alpha$, and $\lambda$ are shown in Figures 15, 16, and 17.

From Figures 12, 13, and 14, we can see that the firm's profit decreases with increasing $T$ or $\lambda$ but increases with increasing $\alpha$. Associated with the relationship between the optimal sales quantity and the three parameters, we find that when the gap 


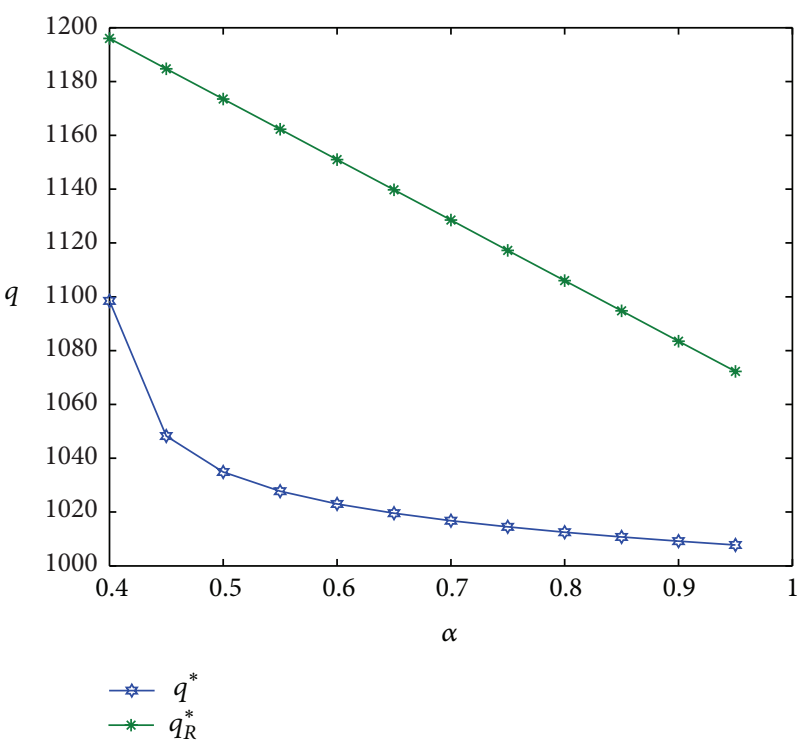

FIGURE 10: Impacts of $\alpha$ on $q^{*}$ and $q_{R}^{*}$.

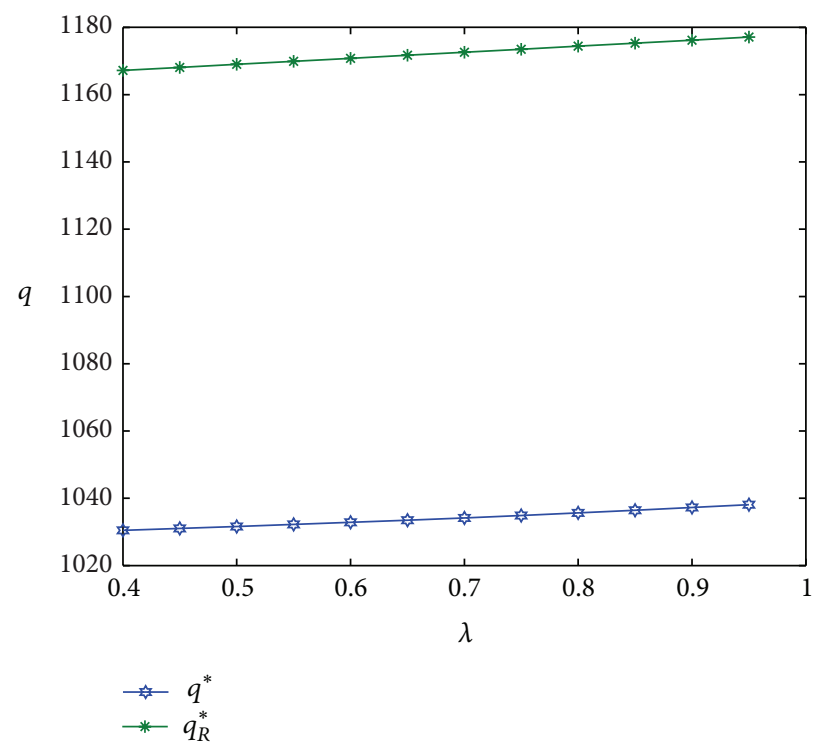

FIgURE 11: Impacts of $\lambda$ on $q^{*}$ and $q_{R}^{*}$.

between the optimal sales quantity and $\mu$ grows larger, profits will fall. Furthermore, we see that when $T, \alpha$, or $\lambda$ is given, the firm's profit under demand uncertainty can reach more than $97 \%$ of that under demand certainty. Therefore, we can draw the conclusion that the maximin approach is not overly conservative.

Figures 15, 16, and 17 show that the money the firm paid for increased information or the loss due to the uncertainty of demand will decrease with increasing $T$ or $\lambda$ and increase with increasing $\alpha$. By comparison and analysis of these three figures, we find that the loss in the second case (only support of the demand is known) is bigger than that in the first case (only $\mu$ and $\sigma$ are known). We can also find that the parameter $\alpha$ has a significant influence on the amount of money the firm

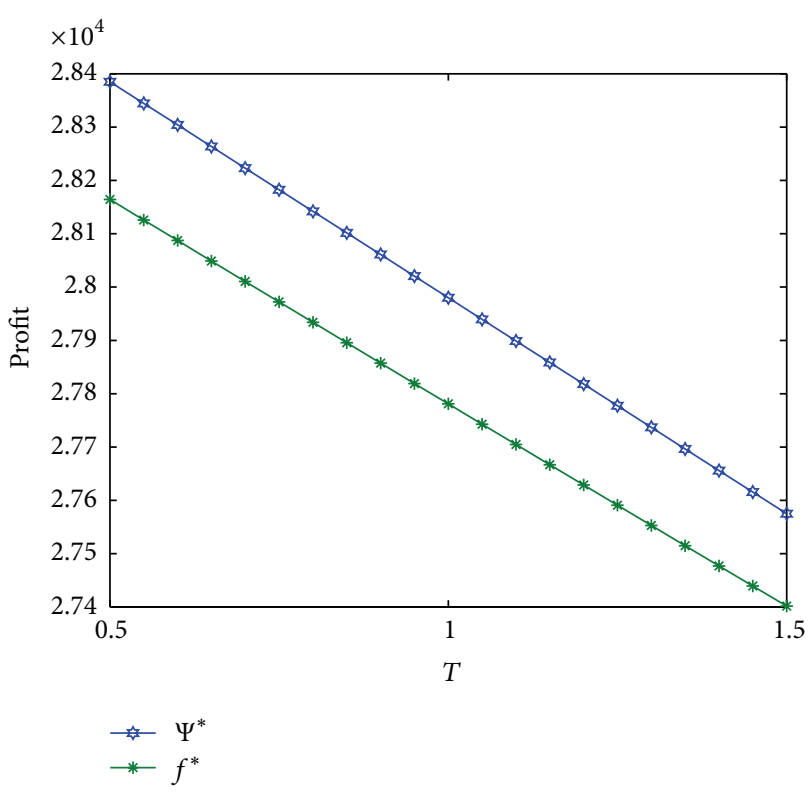

FIgURE 12: Impacts of $T$ on $\Psi^{*}$ and $f^{*}$.

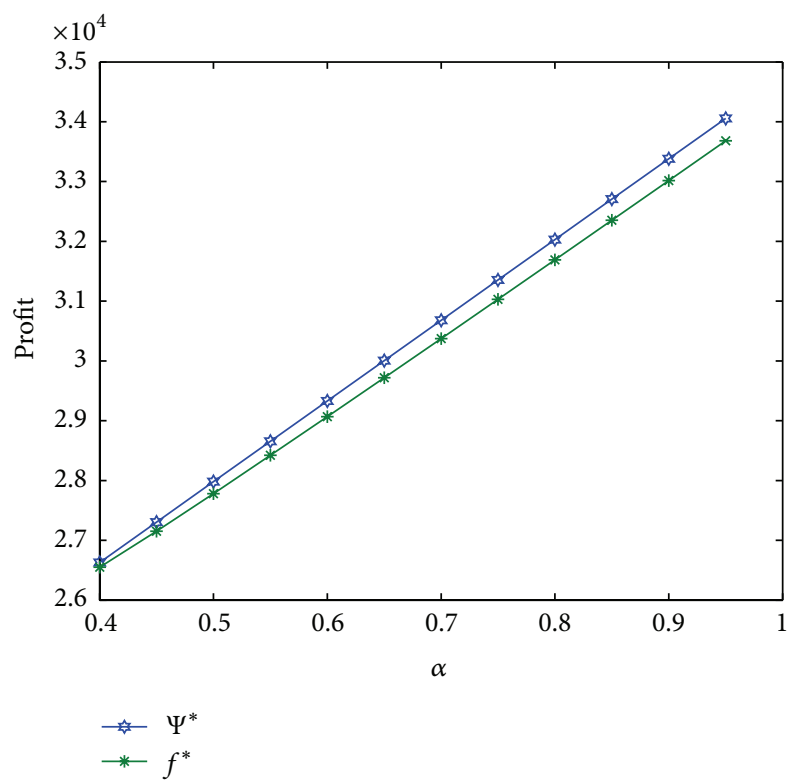

Figure 13: Impacts of $\alpha$ on $\Psi^{*}$ and $f^{*}$.

paid for increased information. Therefore, the firm should invest more on the probability of succeeding in the project.

\section{Conclusion}

In this paper, we use the maximin robust and the minimax regret approach in studying the inventory financing problem, in which a firm having only partial information must make a trade-off between pledging for cash to invest and selling before the sales season. We provided a decision-making aid for the firm and analyzed the impacts of certain parameters and uncertainties upon the firm's decision and profits. We 


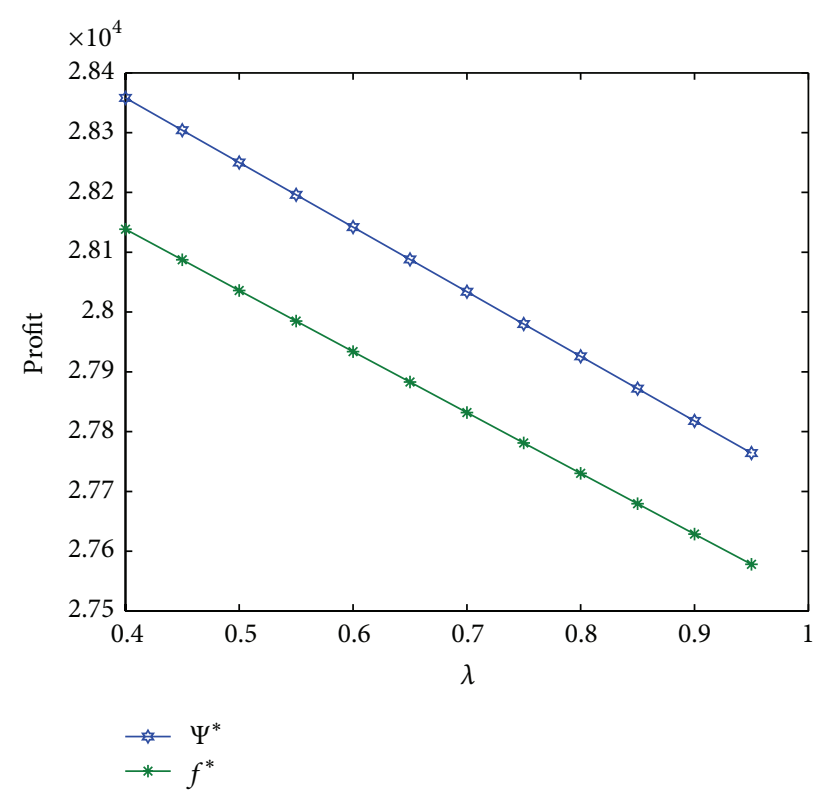

FIgURE 14: Impacts of $\lambda$ on $\Psi^{*}$ and $f^{*}$.

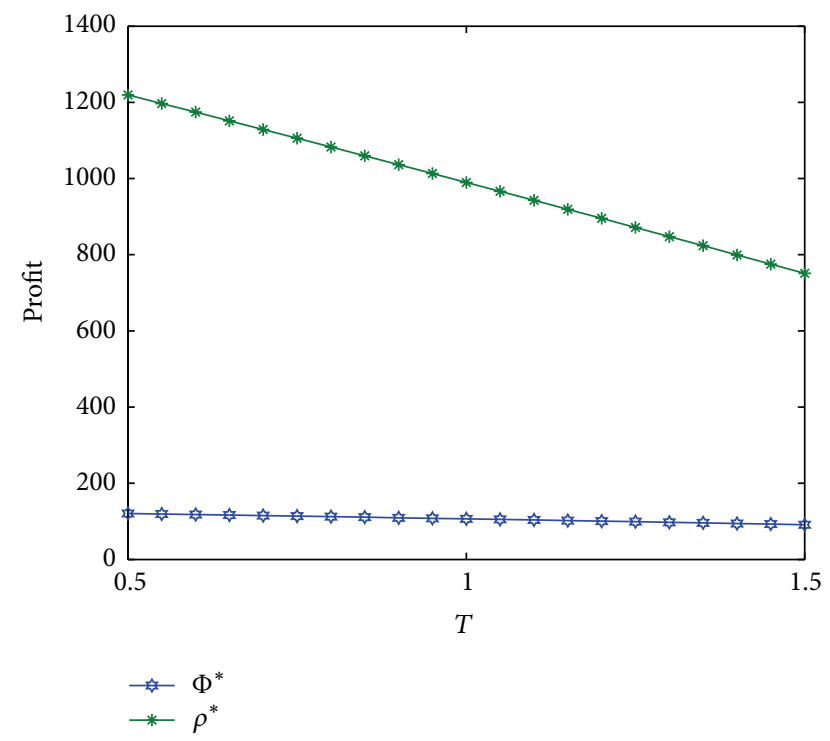

FIgURE 15: Impacts of $T$ on $\Phi^{*}$ and $\rho^{*}$.

obtained new conclusions differing from those in the extant literature. We found that, in the first case, no matter the firm's optimal sales quantity or profit, $\mu$ has much greater impact and the impact of $\sigma$ can be omitted. Accordingly, the firm should invest much more for the information of mean of the demand. In the second case, if the firm only cares about the maximum price it pays to acquire information regarding the exact demand distribution, then it only needs to know the absolute difference between $M$ and $N$. We also found that, in both cases, the firm's optimal sales quantity increases with increasing $T$ or $\lambda$ but decreases with increasing $\alpha$. On the contrary, the firm's profit decreases with increasing $T$ or $\lambda$ and decreases with increasing $\alpha$; that is, if the gap

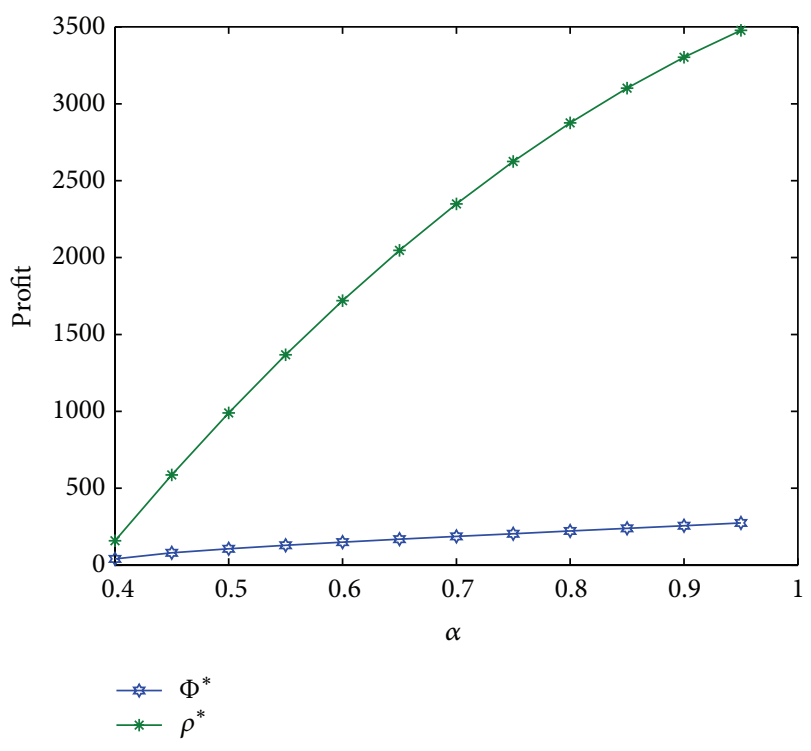

Figure 16: Impacts of $\alpha$ on $\Phi^{*}$ and $\rho^{*}$.

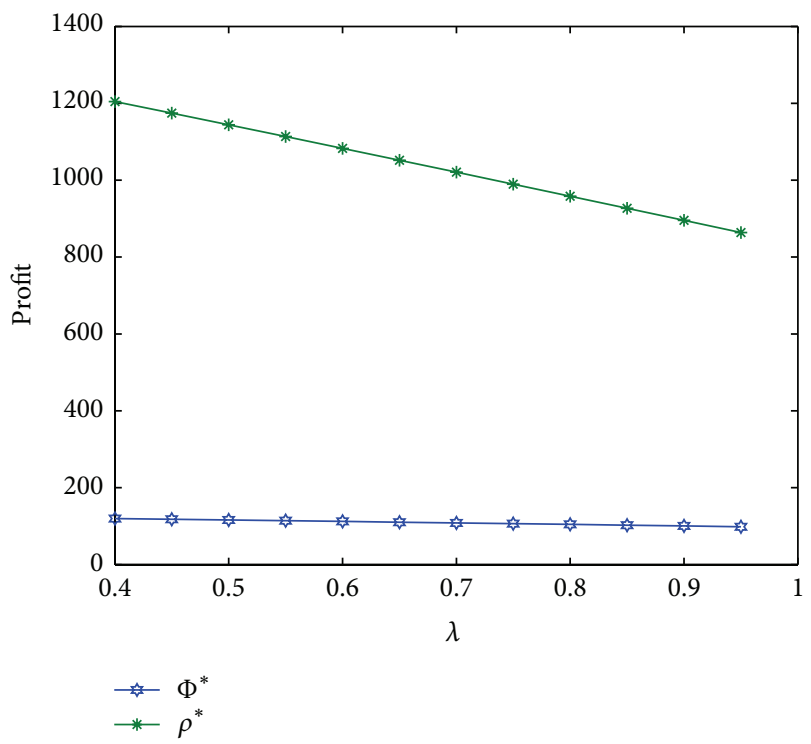

Figure 17: Impacts of $\lambda$ on $\Phi^{*}$ and $\rho^{*}$.

between the optimal sales quantity and $\mu$ grows larger, the profit will fall. We also found that the losses due to demand uncertainty in the second case are bigger than in the first case, a difference that can be attributed to the lower level of available information in the second case. More importantly, we found that the approaches we used here can deal with the problem of partial information well.

Certainly, this paper has limitations. First, our models only study single-period decision problems. It may be interesting to study multiperiod versions in future studies. Second, in this paper, we assumed a fixed selling price for the product. However, the selling price may be a function of demand. Therefore, we can explore the case in which the firm jointly decides both the sales quantity and the selling price. 


\section{Conflict of Interests}

The authors have declared that there is no conflict of interests.

\section{Acknowledgments}

The authors would like to thank the authors of the references for enlightening them. This paper was supported by National Natural Science Foundation of China (71272085), the Humanities and Social Sciences Program of the Ministry of Education of China (12YJA630135).

\section{References}

[1] J. A. Buzacott and R. Q. Zhang, "Inventory management with asset-based financing," Management Science, vol. 50, no. 9, pp. 1274-1292, 2004.

[2] J. Xu and $\mathrm{K} . \mathrm{Fu}$, "Optimal single-period inventory financing decisions with stochastic demand," in Proceedings of the IEEE International Conference on Industrial Engineering and Engineering Management (IEEM '10), pp. 1204-1208, December 2010.

[3] E. Jokivuolle and S. Peura, "Incorporating collateral value uncertainty in loss given default estimates and loan-to-value ratios," European Financial Management, vol. 9, no. 3, pp. 299314, 2003.

[4] D. Cossin, Z. J. Huang, and N. D. Aunon, A Framework for Collateral Risk Control Determination, European Central Bank Working Paper Series, 2003.

[5] V. Babich, G. Aydin, P. Brunet, J. Keppo, and R. Saigal, "Risk, financing and the optimal number of supplier," Working Paper, University of Michigan, 2008.

[6] L. Li, M. Shubik, and M. J. Sobel, "Control of dividends, capital subscriptions, and physical inventories," Working Paper, Weatherhead School of Management, Case Western Reserve University, 2009.

[7] M. Dada and Q. Hu, "Financing newsvendor inventory," Operations Research Letters, vol. 36, no. 5, pp. 569-573, 2008.

[8] M. Q. Suo, Y. P. Li, G. H. Huang, D. L. Deng, and Y. F. $\mathrm{Li}$, "Electric power system planning under uncertainty using inexact inventory nonlinear programming method," Journal of Environmental Informatics, vol. 22, no. 1, pp. 49-67, 2013.

[9] H. Xu, "Managing production and procurement through option contracts in supply chains with random yield," International Journal of Production Economics, vol. 126, no. 2, pp. 306-313, 2010.

[10] K. S. Lari, "A note on baffle orientation in long ponds," Journal of Environmental Informatics, vol. 21, no. 2, pp. 136-141, 2013.

[11] D. Gupta and L. Wang, "A stochastic inventory model with trade credit," Manufacturing \& Service Operations Management, vol. 11, no. 1, pp. 4-18, 2009.

[12] C. H. Lee and B.-D. Rhee, "Channel coordination using product returns for a supply chain with stochastic salvage capacity," European Journal of Operational Research, vol. 177, no. 1, pp. 214238, 2007.

[13] H. Scarf, "A min-max solution of an inventory problem," in Studies in the Mathematical Theory of Inventory and Production, K. Arrow, S. Karlin, and H. Scarf, Eds., chapter 12, pp. 201-209, 1958.
[14] G. Gallego and I. Moon, "The distribution free newsboy problem: review and extensions," Journal of the Operational Research Society, vol. 44, no. 8, pp. 825-834, 1993.

[15] E. S. Mills, "Uncertainty and price theory," The Quarterly Journal of Economics, vol. 73, no. 1, pp. 116-130, 1959.

[16] N. C. Petruzzi and M. Dada, "Pricing and the newsvendor problem: a review with extensions," Operations Research, vol. 47, no. 2, pp. 183-194, 1999.

[17] E. A. Silver and R. Perterson, Decision Systems for Inventory Management and Production Planning, John Wiley \& Sons, New York, NY, USA, 1985.

[18] H. Scarf, "Bayes solutions of the statistical inventory problem," The Annals of Mathematical Statistics, vol. 30, no. 2, pp. 490-508, 1959.

[19] G. A. Godfrey and W. B. Powell, "An adaptive, distribution-free algorithm for the newsvendor problem with censored demands, with applications to inventory and distribution," Management Science, vol. 47, no. 8, pp. 1101-1112, 2001.

[20] R. Levi, R. Roundy, and D. B. Schmoys, "Provably near-optimal sample-based policies for stochastic inventory control models," Cornell Working Paper, 2005.

[21] L. J. Savage, "The theory of statistical decisions," Journal of the American Statistical Association, vol. 46, no. 253, pp. 55-67, 1951.

[22] G. L. Vairaktarakis, "Robust multi-item newsboy models with a budget constraint," International Journal of Production Economics, vol. 66, no. 3, pp. 213-226, 2000.

[23] J. Yue, B. Chen, and M.-C. Wang, "Expected value of distribution information for the newsvendor problem," Operations Research, vol. 54, no. 6, pp. 1128-1136, 2006.

[24] G. Perakis and G. Roeis, "Regret in the newsvendor model with partial information," Operations Research, vol. 56, no. 1, pp. 188203, 2008.

[25] J. Lin and T. S. Ng, "Robust multi-market newsvendor models with interval demand data," European Journal of Operational Research, vol. 212, no. 2, pp. 361-373, 2011.

[26] I. Popescu, "A semidefinite programming approach to optimalmoment bounds for convex classes of distributions," Mathematics of Operations Research, vol. 30, no. 3, pp. 632-657, 2005. 


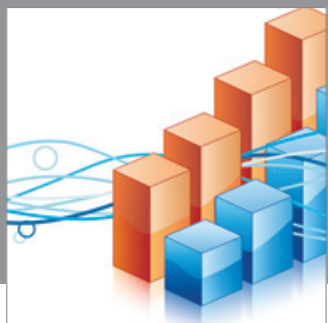

Advances in

Operations Research

mansans

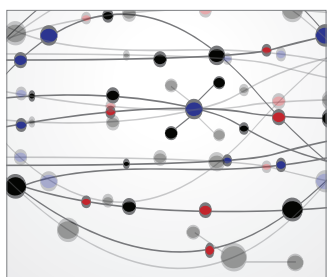

The Scientific World Journal
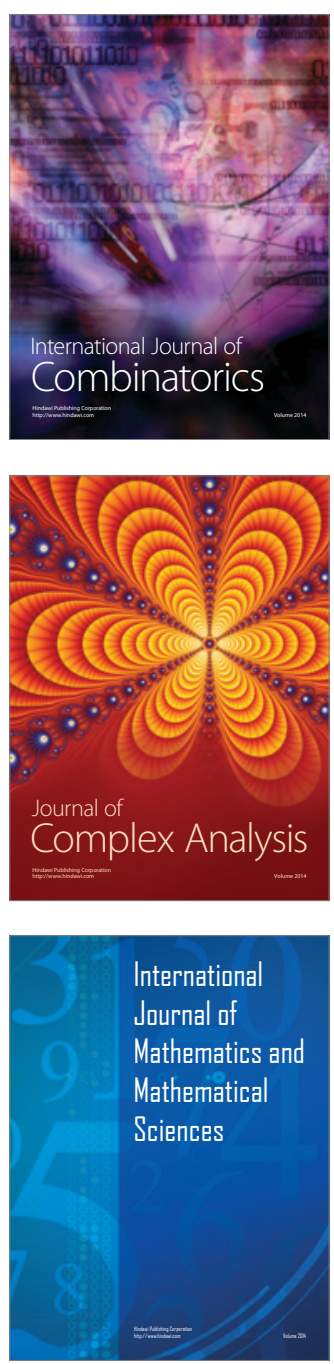
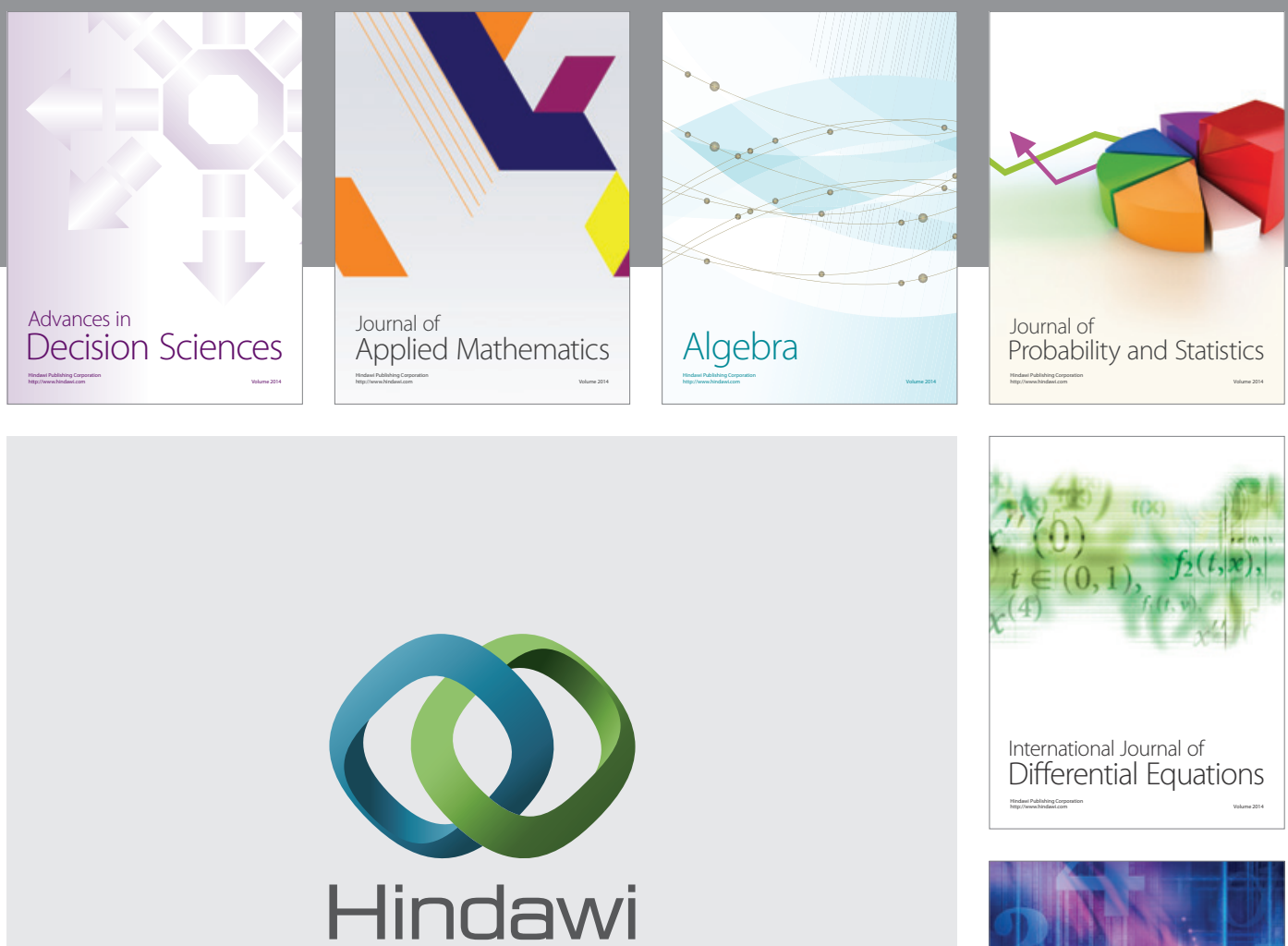

Submit your manuscripts at http://www.hindawi.com
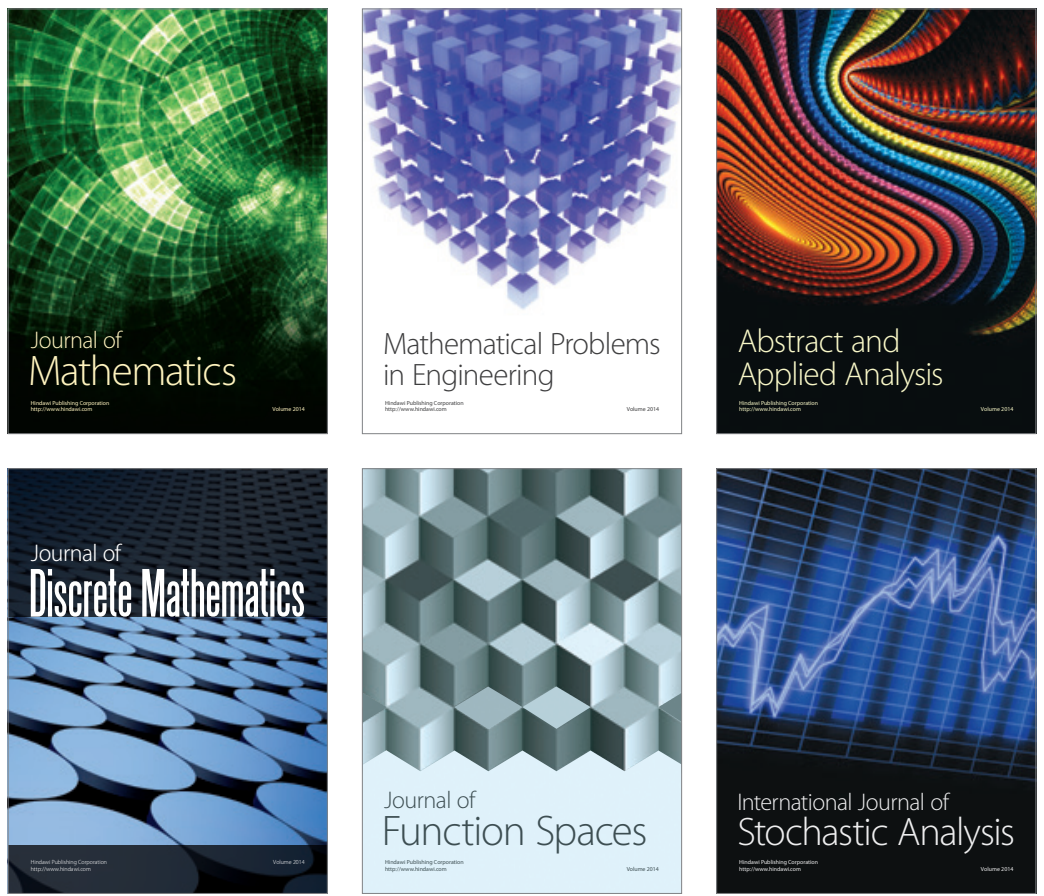

Journal of

Function Spaces

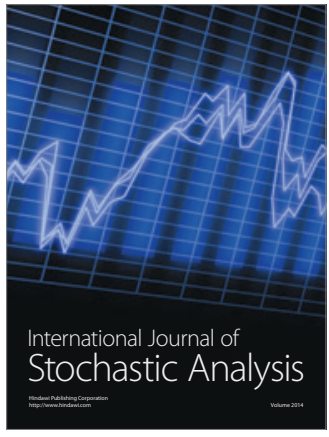

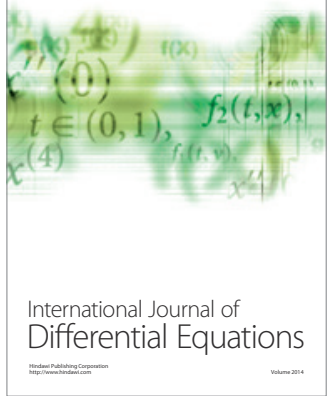
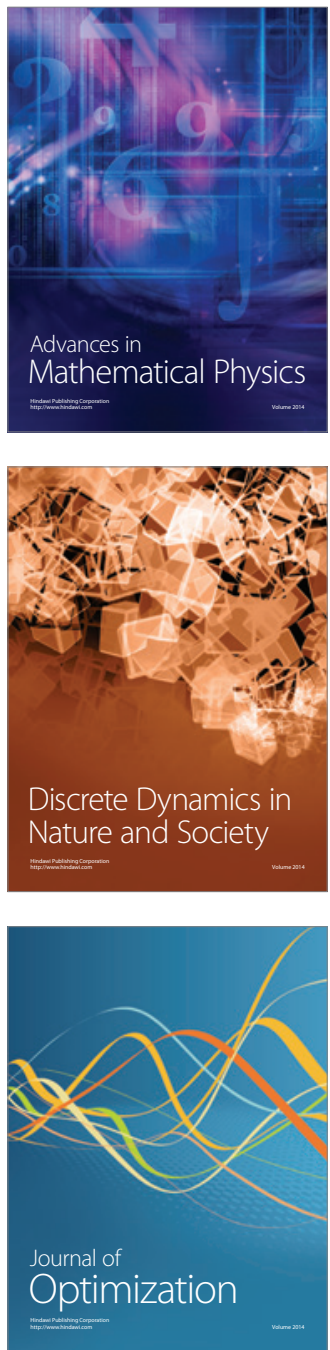